\title{
Lung adenocarcinoma patient with an EGFR kinase domain duplication (KDD) and the response to icotinib
}

\author{
You-Cai Zhu', Wen-Xian Wang ${ }^{2}$, Chun-Wei Xu' ${ }^{3}$, Qing-He Tan ${ }^{4}$, Jian-Ying Li $^{4}$, Wu Zhuang ${ }^{5}$, Zheng-Bo \\ Song $^{2}$, Kai-Qi Du ${ }^{1}$, Gang Chen ${ }^{3}$, Tang-Feng Lv $^{6}$, Yong Song ${ }^{6}$ \\ ${ }^{1}$ Department of Thoracic Disease Diagnosis and Treatment Center, Zhejiang Rongjun Hospital, Jiaxing 314000, China; ${ }^{2}$ Department of \\ Chemotherapy, Zhejiang Cancer Hospital, Hangzhou 310022, China; ${ }^{3}$ Department of Pathology, Fujian Cancer Hospital, Fujian Medical University \\ Cancer Hospital, Fuzhou 350014, China; ${ }^{4}$ Department of Oncology, Nantong Tumor Hospital, Nantong 226361, China; ${ }^{5}$ Department of Medical \\ Thoracic Oncology, Fujian Cancer Hospital, Fujian Medical University Cancer Hospital, Fuzhou 350014, China; ${ }^{6}$ Department of Respiratory \\ Medicine, Jinling Hospital, Nanjing 210002, China \\ Correspondence to: Chun-Wei Xu, MD, PhD. Department of Pathology, Fujian Cancer Hospital, Fujian Medical University, No. 420, Fuma Road, \\ Fuzhou 350014, China. Email: xuchunweibbb@163.com; Wen-Xian Wang, MD, PhD. Department of Chemotherapy, Zhejiang Cancer Hospital, \\ No.1 Banshan East street, Gongshu District, Hangzhou 310022, China. Email: helen-0407@163.com.
}

Submitted Dec 21, 2017. Accepted for publication Apr 19, 2018.

doi: $10.21037 /$ jtd.2018.04.162

View this article at: http://dx.doi.org/10.21037/jtd.2018.04.162

\section{Introduction}

Non-small cell lung cancer (NSCLC) is the leading cause of cancer-related morbidity and mortality worldwide (1). Epidermal growth factor receptor $(E G F R)$ mutations, as driver oncogenes, were first identified in NSCLC in 2004 (2). Approximately $10 \%$ of Caucasian patients and $50 \%$ of Asian NSCLC patients have EGFR mutations $(3,4)$. The most common EGFR mutations are deletions of exon 19, which account for $45 \%$ of all EGFR mutations, and the exon 21-point mutation, L858R, which accounts for $35 \%$ of all EGFR mutations) $(5,6)$. Some clinical trials have demonstrated that most mutations involving exons 18, 19, and 21, especially for sensitive EGFR mutations, are considered predictive of the sensitivity response to EGFR tyrosine kinase inhibitor (TKI) therapy $(5,7,8)$. Due to the limitations of first-generation testing techniques, uncommon EGFR mutations are not detected. It is our opinion that it is uncommon for these wild-type patients to have EGFR mutations. Moreover, these patients have poor survival because they do not receive EGFR-TKIs treatment. With the development of precise detection techniques, more and more rare or atypical EGFR mutations have been identified. Next generation sequencing (NGS) analyses are feasible in patients with NSCLC and identify notable proportions of patients who are potentially eligible for emerging molecular therapeutics, such as exons 18-25 kinase domain duplications (KDDs) and EGFR fusions (EGFR-RAD51 or EGFR-PURB) $(9,10)$. We know EGFRKDD exists in NSCLC patients; however, the efficacy of EGFR-TKI treatment is still uncertain. We report the first case involving the presence of oncogenic EGFR-KDD in China. The patient had stable disease to treatment with an EGFR-TKI. In addition, we have summarized previous cases who had EGFR-KDD mutation NSCLC and whether or not EGFR-TKIs treatment was administered.

\section{Case presentation}

A 63-year-old Chinese female, who was a non-smoker, presented to our hospital for evaluation of a left lung mass on physical examination. A computed tomography (CT) scan showed a mass in the superior lobe of the left lung, and close to a pleural nodule (Figure 1). For staging purposes, surgery was performed. Hematoxylin and eosin staining of the surgical specimen showed a typical morphology for adenocarcinoma cells and surgical specimens from the left lobe tumor and pleural nodes $(2.3 \mathrm{~cm} \times 1.3 \mathrm{~cm}$ $\times 1.0 \mathrm{~cm}$ ) showed lung adenocarcinoma (Figure 2). The immunohistochemical results [TTF-1 (+), NapsinA (+), CK7 (+)] According to the $7^{\text {th }}$ edition of TNM staging, the patient was classified as stage IV (T1N0M1). The patient was wild-type for sensitive $E G F R$ variants, including $E G F R$ 

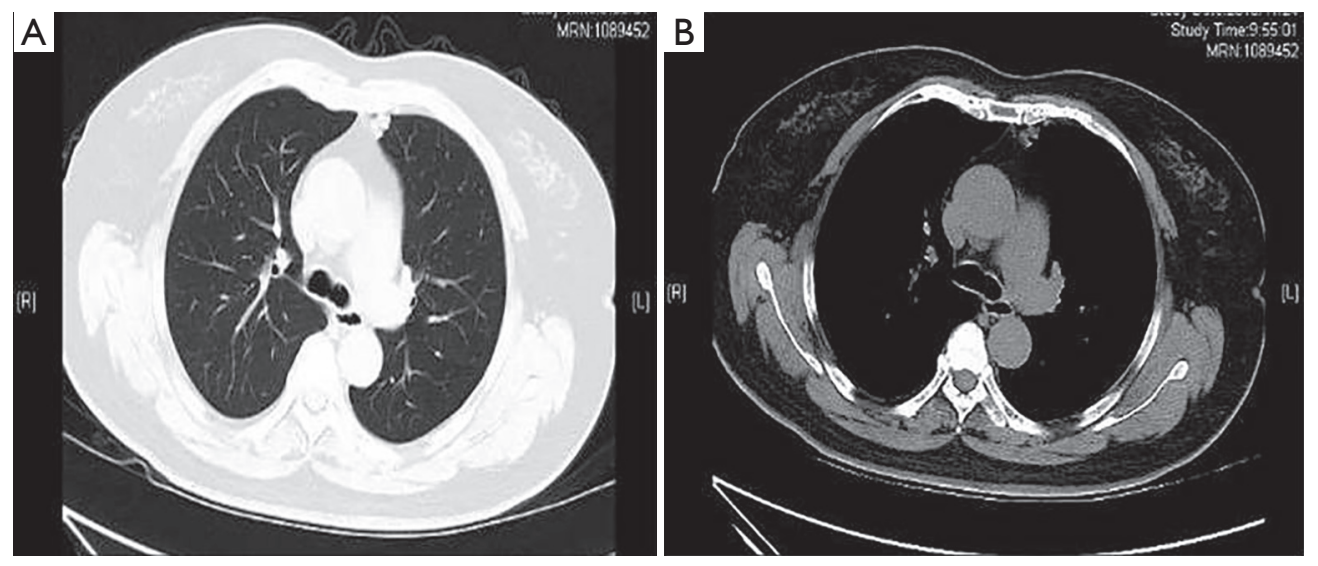

Figure 1 Computed tomography (CT) scans show: (A) lung window before surgery and (B) mediastinal window before surgery.
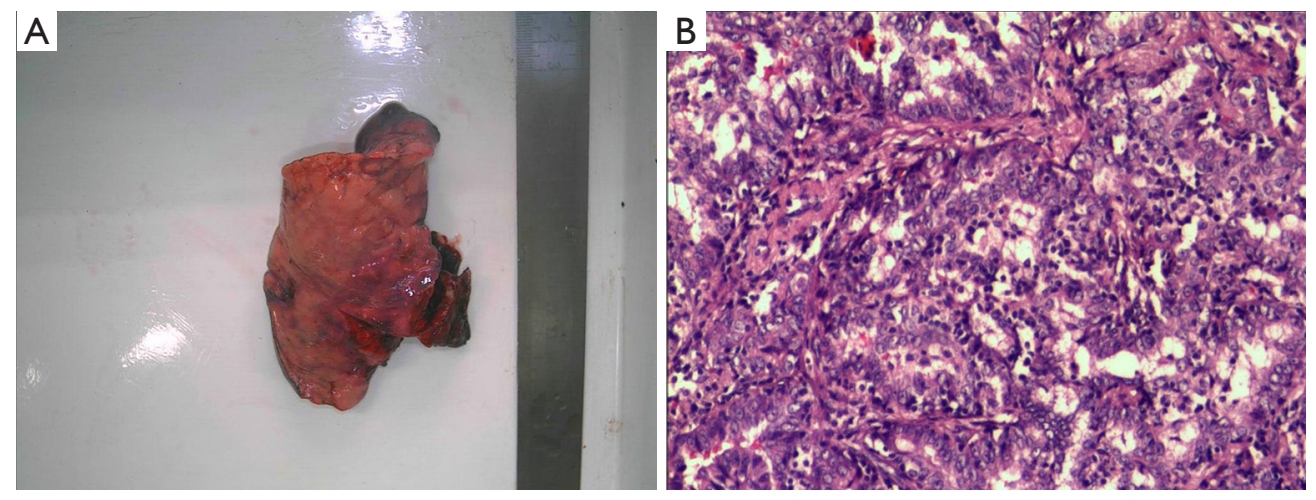

Figure 2 The gross morphology and microscopic morphology of lung tumor. (A) Surgery of left lung tumor; (B) hematoxylin and eosin (H\&E) staining showed a typical morphology for adenocarcinoma cells $(\mathrm{H} \& \mathrm{E}, \times 100)$.

18-21, by ARMS (AmoyDx, Xiamen, China) detection. Using an NGS assay (Illumina, San Diego, CA, USA), we found that the tumor had EGFR-KDD (3D Medicines, Shanghai, China) (Figure 3), and the most common types of EGFR mutations were wild. The patient received icotinib $(125 \mathrm{mg} / \mathrm{tid})$ treatment and showed a stable tumor response according to the Response Evaluation Criteria in Solid Tumors guidelines (version 1.1). During treatment with icotinib, there were no treatment-related adverse events, including hepatic and renal function, gastrointestinal reactions, rashes, and cordis damage. To date, the disease is stable after 11 months and she is continuing treatment with icotinib. Our case is the first report of an EGFRKDD mutation in the Asian population. Considering this rare $E G F R$ mutation and response to TKI treatment, we conclude that the incidence of rare EGFR gene mutations in NSCLC patients should be studied.

\section{Discussion}

EGFR exons 18-25 KDDs were first described in a glioblastoma (11), and now are known to be a driver gene in lung cancer. In 2015, EGFR-KDD was first reported in a patient with lung adenocarcinoma (12). A female patient with metastatic adenocarcinoma, but without a known activating EGFR mutation based on initial polymerase chain reaction testing, underwent a repeat biopsy which was evaluated with a NGS platform. She was shown to have an EGFR-KDD mutation and demonstrated remarkable disease control with erlotinib therapy. In addition, Gallant et al. (9) reported a male patient with lung adenocarcinoma and an EGFR-KDD mutation detected with NGS. The patient had a partial response to afatinib therapy. These findings were studied in vitro and EGFR-KDD expression had significantly increased colony formation compared to 


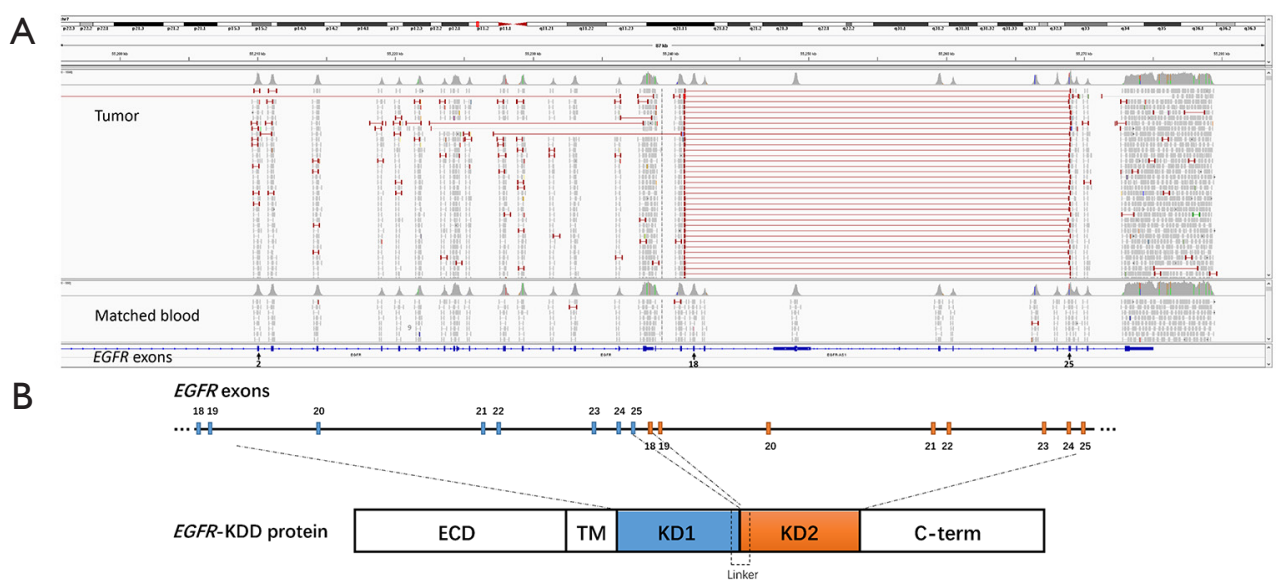

Figure 3 The EGFR-KDD is an oncogenic EGFR alteration. (A) the Integrative Genomics Viewer snapshot of paired NGS reads of tumor and matched blood; (B) schematic representation of EGFR-KDD depicting the genetic and protein domain structures. Blue, EGFR exons 18-25 \#1; orange, EGFR exons 18-25 \#2. EGFR, epidermal growth factor receptor; KDD, kinase domain duplication; ECD, extracellular domain; TM, transmembrane domain; KD1, first kinase domain; KD2, second kinase domain; C-term, carboxyl terminus.

the EGFR wild type and oncogenic EGFR-L858R mutation. Therefore, EGFR-KDD is an oncogenic alteration. Gallant et al. (9) also analyzed whether or not EGFR-TKIs is an effective therapeutic strategy for tumors harboring the EGFR-KDD mutation. Third-generation EGFR-TKIs (erlotinib, afatinib, and AZD9291) are able to inhibit EGFR-KDD tyrosine phosphorylation in a dose-dependent manner, albeit to different levels, and afatinib is the most potent inhibitor of EGFR-KDD autophosphorylation to decrease cell viability. The current report describes the first Asian patient with an EGFR-KDD mutation who derived a sustained anti-tumor response from treatment with the EGFR-TKI, icotinib. We have summarized the lung cancer patients who have been reported to have EGFR-KDD mutations in Table 1. Therefore, for this type mutation in NSCLC patients, EGFR inhibitors may be a first-line therapy, as in sensitive EGFR mutation patients.

A majority of patients ultimately develop acquired resistance to EGFR-TKI therapy and experience disease progression with a median progression-free survival of 9-13 months $(5,7,8)$. With the in-depth study of resistance mechanisms, researchers have found various resistance mechanisms to first-generation EGFR-TKIs. The EGFR T790M mutation is regarded as the most common cause of acquired resistance, affecting $50 \%$ of patients (13). Other mechanisms include bypass signaling activation, such as MET gene amplification, BRAF (G469A and V600E) mutations, and HER2 amplification and phenotype transition, including transition to small cell lung cancer and epithelial mesenchymal transition (14). Whether or not the acquired resistance mechanisms of EGFR-TKIs to uncommon EGFR mutation is similar to sensitive mutations is unclear. In the case described by Gallant et al. (9), the patient develop acquired resistance to afatinib after seven cycles of therapy. Molecular profiling was performed on the afatinib-resistant tumor biopsy sample, which showed significant amplification of the EGFR-KDD allele that differed from his pre-treatment tumor sample. Amplification of the mutant EGFR allele has been reported as a mechanism of acquired resistance in lung cancer patients with sensitive EGFR mutations (15). In addition, in the case reported by Christina S. Baik, the post-TKI resistance biopsy demonstrated a low level of T790M (12). Therefore, the acquired resistance of EGFR-KDD to EGFR-TKI may include the most common mechanisms of T790M mutation (a sensitive gene mutation, amplification of the EGFR-KDD mutation, and an EGFR-dependent mechanism of resistance). Due to the small number of cases, further exploring therapeutic targets in these patients and acquired resistance mechanisms are needed.

The polymerase chain reaction-based testing method is the standard assay routinely used clinically for NSCLC patients; however, some rare or uncommon types of EGFR gene alterations are not identified. Developments in multiple genome sequencing, such as NGS, is a more precise method for detecting a large number of gene alterations with known 
Table 1 Clinical characteristics of patients with non-small cell lung cancer harboring EGFR KDD

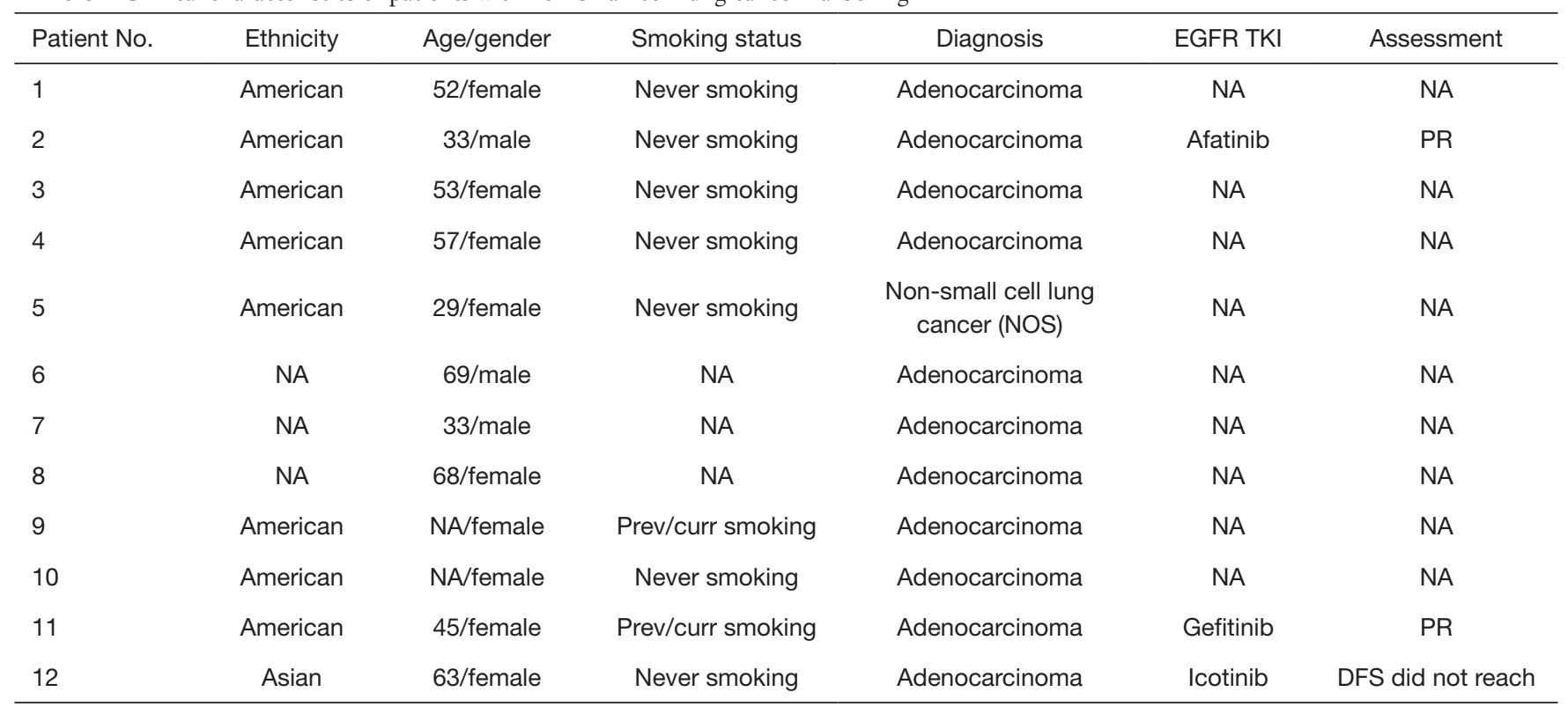

EGFR, epidermal growth factor receptor; KDD, kinase domain duplication; TKI, tyrosine kinase inhibitor; PR, partial response; DFS, disease-free survival.

and unknown gene mutations (16). Moreover, NGS can provide information for resistance mechanisms after disease progression on targeted therapy. For lung cancer patients to benefit from more personalized cancer treatment, clinical therapy should improve with clinical diagnostics through multi-gene assays to determine the actual clinical benefits.

This case report increases the evidence supporting EGFR-TKI treatment of EGFR-KDD mutations in NSCLC patients. In the future, we think it is possible to discover rare gene mutations during the routine use of multi-gene sequencing assays and explore differences in the acquired mechanism of $1^{\text {st }}-3^{\text {rd }}$ generation EGFR inhibitors between sensitive EGFR mutations and other uncommon mutations in NSCLC patients.

\section{Acknowledgements}

Funding: This study was supported in part by grants from the Science and Technology Planning Project of Zhejiang Province (2015C33194) and the National Clinical Key Specialty Construction Program (2013) supported this work.

\section{Footnote}

Conflicts of Interest: The authors have no conflicts of interest to declare.
Informed Consent: Written informed consent was obtained from the patient for publication of this manuscript and any accompanying images.

\section{References}

1. Siegel RL, Miller KD, Jemal A. Cancer Statistics, 2017. CA Cancer J Clin 2017;67:7-30.

2. Lynch TJ, Bell DW, Sordella R, et al. Activating mutations in the epidermal growth factor receptor underlying responsiveness of non-small-cell lung cancer to gefitinib. N Engl J Med 2004;350:2129-39.

3. Shi $\mathrm{Y}, \mathrm{Au} \mathrm{JS}$, Thongprasert $\mathrm{S}$, et al. A prospective, molecular epidemiology study of EGFR mutations in Asian patients with advanced non-small-cell lung cancer of adenocarcinoma histology (PIONEER). J Thorac Oncol 2014;9:154-62.

4. Hirsch FR, Bunn PA Jr. EGFR testing in lung cancer is ready for prime time. Lancet Oncol 2009;10:432-3.

5. Mok TS, Wu YL, Thongprasert S, et al. Gefitinib or carboplatin-paclitaxel in pulmonary adenocarcinoma. $\mathrm{N}$ Engl J Med 2009;361:947-57.

6. Maemondo M, Inoue A, Kobayashi K, et al. Gefitinib or chemotherapy for non-small-cell lung cancer with mutated EGFR. N Engl J Med 2010;362:2380-8.

7. Rosell R, Carcereny E, Gervais R, et al. Erlotinib 
versus standard chemotherapy as first-line treatment for European patients with advanced EGFR mutation-positive non-small-cell lung cancer (EURTAC): a multicentre, open-label, randomized phase 3 trial. Lancet Oncol 2012;13:239-46.

8. Shi Y, Zhang L, Liu X, et al. Icotinib versus gefitinib in previously treated advanced non-small-cell lung cancer (ICOGEN): a randomised, double-blind phase 3 noninferiority trial. Lancet Oncol 2013;14:953-61.

9. Gallant JN, Sheehan JH, Shaver TM, et al. EGFR kinase domain duplication (EGFR-KDD) is a novel oncogenic driver in lung cancer that is clinically responsive to afatinib. Cancer Discov 2015;5:1155-63.

10. Konduri K, Gallant JN, Chae YK, et al. EGFR fusions as novel therapeutic targets in lung cancer. Cancer Discov 2016;6:601-11.

11. Ozer BH, Wiepz GJ, Bertics PJ. Activity and cellular localization of an oncogenic glioblastoma multiformeassociated EGF receptor mutant possessing a duplicated kinase domain. Oncogene 2010;29:855-64.

12. Baik CS, Wu D, Smith C, et al. Durable Response to

Cite this article as: Zhu YC, Wang WX, Xu CW, Tan QH, Li JY, Zhuang W, Song ZB, Du KQ, Chen G, Lv TF, Song Y. Lung adenocarcinoma patient with an EGFR kinase domain duplication (KDD) and the response to icotinib. J Thorac Dis 2018;10(5):E359-E363. doi: 10.21037/jtd.2018.04.162
Tyrosine Kinase Inhibitor Therapy in a Lung Cancer Patient Harboring Epidermal Growth Factor Receptor Tandem Kinase Domain Duplication. J Thorac Oncol 2015;10:e97-9.

13. Oxnard GR, Arcila ME, Chmielecki J, et al. New strategies in overcoming acquired resistance to epidermal growth factor receptor tyrosine kinase inhibitors in lung cancer. Clin Cancer Res 2011;17:5530-7.

14. Zhong WZ, Zhou Q, Wu YL. The resistance mechanisms and treatment strategies for EGFR-mutant advanced nonsmall-cell lung cancer. Oncotarget 2017;8:71358-70.

15. Sequist LV, Waltman BA, Dias-Santagata D, et al. Genotypic and histological evolution of lung cancers acquiring resistance to EGFR inhibitors. Sci Transl Med 2011,3:75ra26.

16. Lindquist KE, Karlsson A, Levéen P, et al. Clinical framework for next generation sequencing based analysis of treatment predictive mutations and multiplexed gene fusion detection in non-small cell lung cancer. Oncotarget 2017;8:34796-810. 